\title{
Use of video education in postoperative patient counselling: A quality improvement initiative
}

\author{
Luke D.E. Witherspoon, MD'; Ailsa M.L. Gan, MD'; Rodney H. Breau, MD',2; Ginette Saumure, RN; \\ Jacqueline Shea, RN, MScN3; Ranjeeta Mallick, MD'; Jeffrey E. Warren, MD'; Brian Blew, MD'; llias \\ Cagiannos, $M D^{1}$; Christopher Morash, $M D^{1}$; Luke T. Lavallée, $M D^{1,2}$
}

'Division of Urology, Department of Surgery, The Ottawa Hospital and University of Ottawa, Ottawa, ON, Canada; ${ }^{2}$ Ottawa Hospital Research Institute, Ottawa, ON, Canada; ${ }^{3 T h e}$ Ottawa Hospital, Ottawa, ON, Canada

\begin{abstract}
Cite as Witherspoon LDE, Gan AML, Breau RH, et al. Use of video education in postoperative patient counselling: A quality improvement initiative. Can Urol Assoc J 2021;15(12):E658-63. http://dx.doi.org/10.5489/cuaj.7240
\end{abstract}

Published online June 22, 2021

Appendix available at cuaj.ca

\section{Abstract}

Introduction: This quality improvement study examined if a video-based resource could reduce delayed discharges after robotic prostatectomy while maintaining high levels of patient satisfaction. Methods: From April 2018 to February 2020, all patients undergoing robotic-assisted radical prostatectomy (RARP) were asked to complete an anonymous survey evaluating their perioperative experience. The quality improvement (QI) intervention started in March 2019 with a series of six educational videos being shown to all patients. The videos were used to supplement postoperative instruction. The discharge times of all patients were obtained from The Ottawa Hospital Data Repositories. A run chart analysis was used to detect change in discharge time (outcome measure). Patient satisfaction (balancing measure) was analyzed using Chi-squared analysis and descriptive statistics.

Results: A total of 425 robotic prostatectomies (199 pre-intervention, 226 post-intervention) were available. Analysis of the run chart revealed non-random change favoring earlier discharge in the intervention group $(\mathrm{p}<0.05)$, with a pre-intervention late discharge rate of $64 \%$ and a post-intervention late discharge rate of $55 \%$. A total of 140 surveys (59 pre-intervention, 81 post-intervention) assessing patient satisfaction were completed, corresponding with a response rate of $29.6 \%$ and $35.8 \%$, respectively. Median score on a 10-point scale for overall satisfaction was equal between the intervention and non-intervention groups (9 [interquartile range (IQR 8-10) vs. 10 [IQR 8-10], p=0.92).

Conclusions: Patient satisfaction with care and education was high for all patients and was not negatively impacted by this intervention. Video education tools may be one method to help improve the discharge process following RARP.

\section{Introduction}

The surgical approach to radical prostatectomy has changed over the last two decades from being a traditionally open surgical procedure, to being performed laparoscopically, and now the majority are performed via robotic-assisted surgical approaches. ${ }^{1,2}$

One barrier to more widespread adoption of robotic surgery is cost. ${ }^{3-5}$ One of the best-defined benefits of the robotic approach has been a reduction in the length of hospital stay (LOS), which helps offset costs of robotic technology. ${ }^{6-9}$ Continued effort towards reducing the costs associated with this procedure must be sought to make its use sustainable for healthcare systems.

One method to reduce cost is improving the discharge process to help ensure timely and safe discharge from hospital. In one analysis of over 150 quality improvement (QI) plans submitted by hospitals within Ontario, Canada, patient experience was among the most popular choices for the highest priority topic. ${ }^{10}$ Specific desired areas for improvement included communication to patients and streamlining of admission, transfer, and discharge processes. ${ }^{10}$ Unnecessary variation in the discharge process has been found to delay discharge times and decrease quality of care. ${ }^{11}$ Patient instruction and education for postoperative recovery and care requirements is classically given by verbal and/or written instruction by multiple members of the care team, such as nurses and physicians. ${ }^{12}$ Receipt of this information is an imperative milestone in achieving preparedness for discharge. ${ }^{13}$ Additionally, patient understanding of their condition and expectations for treatment and management highly correlate with patient satisfaction and decreased postoperative complications. ${ }^{14,15}$ Although not studied to date, it is likely that given the non-standardized verbal nature of the majority of this teaching, there is highly variable instruction/education received by the patients.

Patients undergoing robotic-assisted radical prostatectomy (RARP) are expected to have a short hospital stay postoperatively, and barring any immediate complications, discharge 
is routinely achievable on postoperative day $1 .{ }^{16}$ Root cause analysis by the clinical care team (nursing, clinical care manager, physician team) identified that the time demand on nursing staff, who have a high volume of time-sensitive clinical tasks for several patients at once, and patient anxiety around preparedness may be possible contributing factors to delayed discharges.

In this QI study, we sought to improve efficiency of the discharge process and standardization of the delivery of patient education prior to discharge for patients undergoing RARP by implementing a video tool that would provide pertinent information to patients prior to discharge. We hypothesized that this transfer of responsibility from nursing and physician staff to the video tool would allow care to be directed towards other daily tasks, allowing earlier average discharges to be achieved.

\section{Methods}

\section{Study cohort and design}

All patients undergoing RARP at The Ottawa Hospital (Ottawa, Canada) between April 2018 and March 2020 were included. Patients undergoing RARP between March 26, 2019 and March 2020 were given the video education tool. The primary outcome was discharge time.

\section{Intervention development}

Within the study center, typical educational activities surrounding RARP include preoperative counselling by the primary surgeon and optional educational classes led by a specialized nurse. Postoperatively, patients are counselled by their nursing team concerning the correct care of surgical wounds and Foley catheter maintenance, while resident physicians provide instruction surrounding expected recovery and followup prior to discharge.

A series of six educational videos were created to encompass the routine teaching given to each patient following RARP. The nursing educational content of these videos was created following multidisciplinary discussion between nurse educators and managers and was based on best nursing practices. ${ }^{17}$ The physician education components were created after consultation with all surgeons performing the procedure at The Ottawa Hospital. The three videos detailing physician education topics were hosted by a resident physician, while the nursing education videos were hosted by a team of nursing staff. Following creation of draft educational scripts, the language of the content was reviewed by The Ottawa Hospital's Patient Education Department for ease of understanding and transcribed into both English and French at a grade-school level. The length of each video was kept to 1.5-3 minutes. Topics covered in the instructional videos included proper Foley catheter and wound care, reasons to seek urgent medical attention, optimal approaches to pain management, and description of the followup process.

In the intervention group, the patients were given an $\mathrm{iPad}^{\mathrm{Tm}}$ upon which they could access the video series, and wherein patients could control the video playback, allowing them to pause and replay. Once patients had viewed all education videos, nursing staff returned and offered to clarify any patient concerns. The outcome measure was discharge times, which were obtained from The Ottawa Hospital Data Repositories derived directly from the electronic medical record. Discharge time was categorized into early (00:00-11:00), midday (11:01-14:00), delayed (14:01-17:00), and very delayed (17:01-23:59). Early and midday discharges were agreed upon as targeted discharge times, as this reflected nursing and hospital policy in place prior to the intervention.

The process measure was fidelity of the intervention; "completion of iPad ${ }^{\mathrm{TM}}$ video teaching" was added to the discharge criteria checklist required for approval by the clinical care manager on the ward prior to discharge for patients in the intervention group. The balancing measure was patient satisfaction.

Data were collected prospectively in the form of voluntary surveys, available in both English and French, given to patients by nursing staff and completed immediately prior to discharge. There are no currently available established postoperative patient experience questionnaires validated in this population. As a result, patient satisfaction scores were based on 5- and 10-point Likert scales evaluating the perioperative experience for both the pre- and post-intervention groups through several targeted questions (Appendix; available at cuaj.ca).

Questions included patient-reported satisfaction surrounding pre-surgery teaching, post-surgery pain control, post-surgery assessments by physicians/nursing staff, and quality of hospital facilities. Questions pertaining to discharge education included patient-reported satisfaction surrounding physician and nursing discharge instructions, anxiety surrounding discharge, and an overall rating of the patients experience while in hospital. Patients were able to specifically indicate if they had concerns about different areas surrounding discharge instructions, including how to perform personal care, take medications at home, or how followup was being arranged. Patients were specifically questioned as to whether they were provided with a plan for their Foley catheter and wound dressings. For the patient cohort receiving educational videos, they were asked to rank the usefulness of these videos on a 10-point scale from not useful to very useful.

\section{Process measure}

Fidelity, as measured by completion of the instructional videos, was $100 \%$ for the duration of the study period as recorded by the clinical care manager. 


\section{Data analysis}

A run chart was used to display the mean monthly discharge times (outcome measure) before and after the intervention. The outcome measure selected was discharge time, aiming to reduce the number of delayed discharges (defined as after $2 \mathrm{pm}$ on day of discharge) to $<25 \%$ by April 1, 2020 . The balancing measure studied was patient satisfaction and experience after educational video introduction, aiming to show non-inferiority to in-person, verbal instruction only.

This approach was selected to demonstrate non-random evidence of change and sustainability. Additionally, we aggregated the outcome data into pre- and post-intervention cohorts and performed a Chi-squared test to assess for statistically significant difference in mean discharge time. Descriptive statistics were used to evaluate fidelity (process measure). Patient satisfaction and experience (balancing measure) was analyzed using Student's t-test and Chi-squared analysis where appropriate. The purpose of the balancing measure is to determine if perceived quality of care and satisfaction levels suffer with the introduction of the video tool as the primary modality of education, as opposed to in-person, verbal instruction alone.

Institutional ethics board approval was obtained for this study. SAS software version 9.4 for Windows was used for the analyses (Cary, NC, U.S.).

\section{Results}

\section{Study population}

During the study period, a total of 425 patients (199 preintervention vs. 226 post-intervention) underwent RARP, all of which had discharge times recorded. The study was ended in February 2020. Baseline characteristics of surveyed men revealed a mean overall age of 64.1 (standard deviation [SD] 7.0) years, with a pre-intervention mean age of 64.1 (SD 7.5) years, and a mean post intervention age of 64.2 (SD 6.8) years.

\section{Outcome measure}

The proportion of delayed discharges decreased during the study period (Fig. 1), from a mean of $62 \%$ pre-intervention to $55 \%$ post-intervention. The median monthly percentage of delayed discharges across the study period was $61 \%$. Interpretation of the run chart, using probability-based rules based on a $\alpha$ error of $p<0.05,{ }^{18}$ revealed two rules were met to suggest significant change: 1) a shift towards improvement is observed after implementation of the intervention; and 2) a non-random pattern is signalled by the existence of too few runs (crossings of the median line). The mean discharge time pre-intervention was 14:40 (SD 2.6) hours; the mean post-intervention discharge time was 14:00 (SD 2.1) hours. When assessing by the pre-defined discharged windows (early, midday, delayed, very delayed), there were fewer dis-

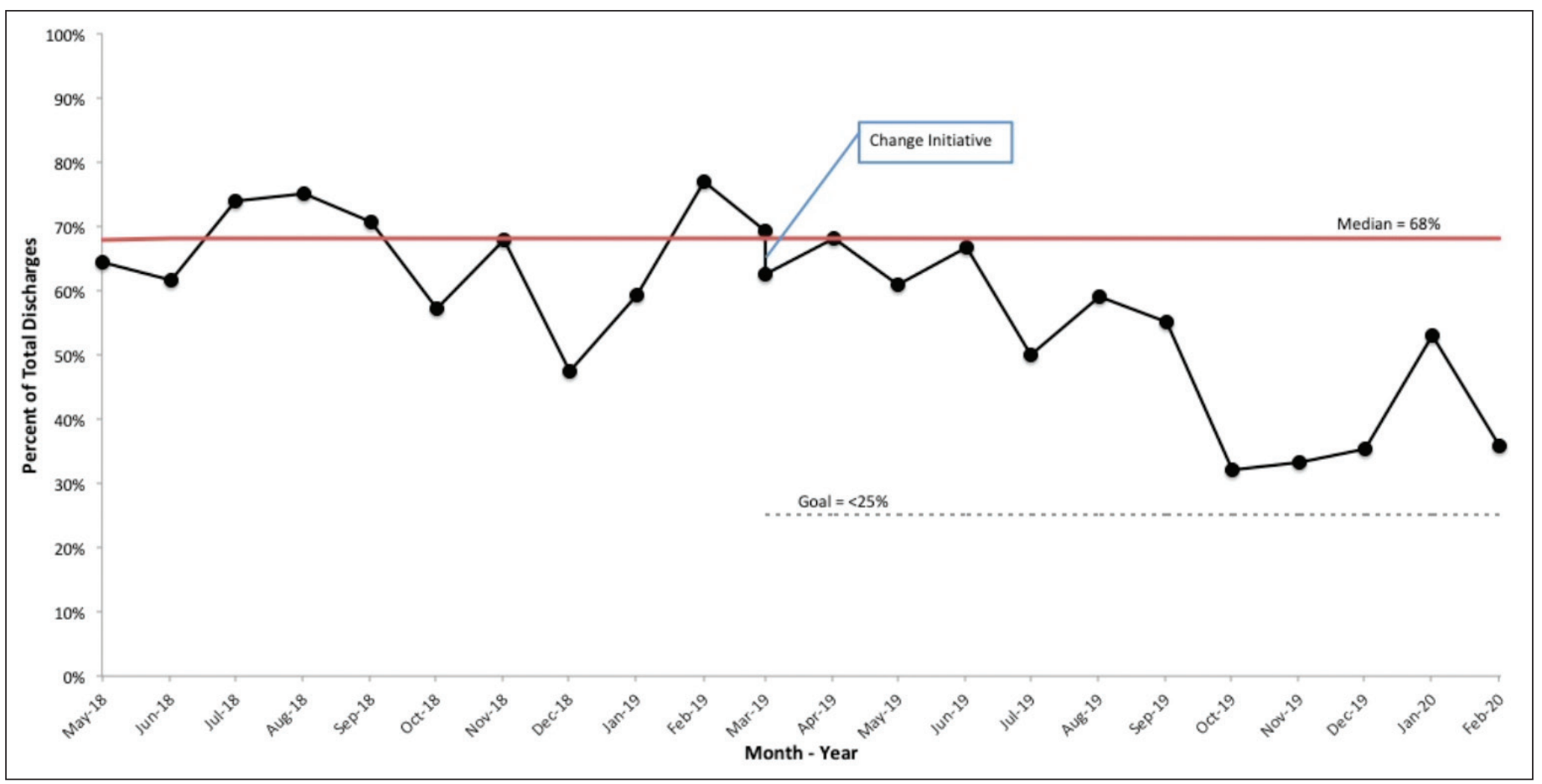

Fig. 1. Run chart depiction of discharge time before and after educational intervention. 


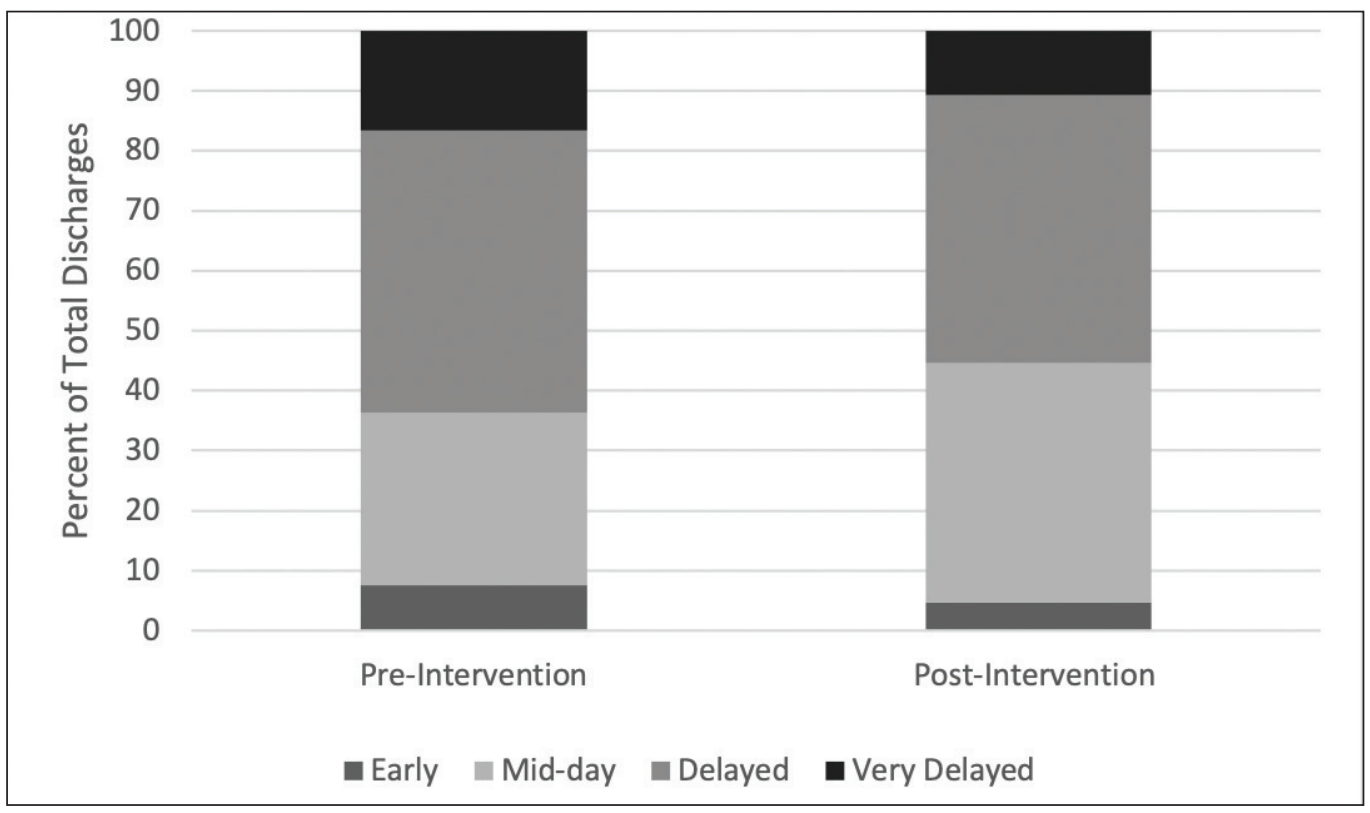

Fig. 2. Assessment of discharge time window pre- and post-video education intervention implementation.

charges in the early window post-intervention (5\% of total discharges) then pre-intervention ( $8 \%$ of total discharges). Midday discharges increased post-intervention ( $40 \%$ of total discharges) as compared to pre-intervention (29\% of total discharges). Delayed discharges decreased post-intervention ( $45 \%$ of total discharges) vs. pre-intervention $(47 \%$ of total discharges). Very delayed discharges further decreased postintervention ( $11 \%$ of total discharges) compared to pre-intervention (17\% of total discharges) (Fig. 2).

\section{Balancing measure}

Pre-intervention survey response rates were $29.6 \%$, with post-intervention response rates of $35.8 \%$. When assessing patient reported mean satisfaction pre- and post-intervention, no statistical differences were noted in reported post-surgery pain (pre-intervention 4.3 [SD 1.0], post-intervention 4.35 [SD 1.1]), assessment by physician team (pre-intervention 4.32 [SD 1.0], post-intervention 4.38 [SD 1.0]), assessment by nursing team (pre-intervention 4.4 [SD 1.0], post-intervention 4.5 [SD 1.1]), hospital facilities (pre-intervention 4.3 [SD 1.0], post-intervention 4.5 [SD 1.1]), or discharge instructions (pre-intervention 4.4 [SD 1.0], post-intervention 4.2 [SD 1.1]) (Fig. 3).

Patients undergoing the education video intervention ranked the usefulness of the videos on a 10-point scale as 8.4 (SD 1.6), and 58\% indicated they would like to see more video educational tools used within the postoperative phase of care.

Furthermore, comparison of mean patient-reported anxiety (pre-intervention 3.0 [SD 2.18], post-intervention 3.1 ]SD 2.2]) and mean overall hospital experience (pre-intervention
8.6 [SD 2.2], post-intervention 9.1 [SD 1.0]) scores did not significantly differ pre- and post- intervention.

\section{Discussion}

\section{Main findings}

This study found that use of a video instructional platform can be one tool to help improve the discharge process after robotic prostatectomy, while maintaining high levels of patient satisfaction. This study did not remove all physician/ nursing in-person patient education, but rather shifted the responsibility of education from in-person sessions to video education tools, supplemented by in-person followup prior to discharge.

Following implementation of our intervention, we identified a trend toward earlier discharge times for our patients, with fewer patients having delayed discharge times, suggesting that this tool may have helped standardize the discharge process. This change took time to see improvement, suggesting that it may have taken time for nursing and physician teams to adapt to the improved efficiency of patient education that these videos may provide, and adjust their own workflow accordingly. Taken together, these findings support the ongoing use of this tool in postoperative teaching to help augment our patient's understanding of their recovery while simultaneously reducing the workload for physicians and nurses. 


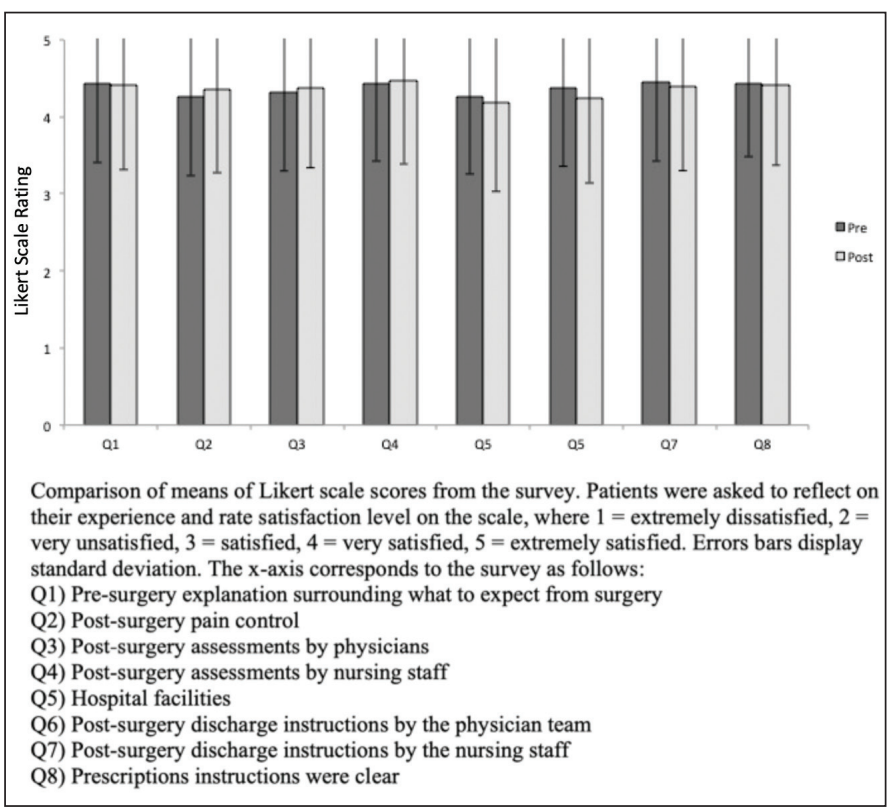

Fig. 3. Patient satisfaction before and after educational video intervention.

\section{Interpretation and comparison to other studies}

Postoperative education is an integral part of the discharge process. Effective postoperative teaching has been associated with decreased re-admission, morbidity, mortality, and hospital costs. ${ }^{12,19,20}$ Patient experience is closely tied to education, with symptoms and anxiety being associated with poor education in both those in the postoperative period and those with non-surgical medical conditions. ${ }^{21-23}$ However, there are no currently established, standardized, postoperative education regimes for our patients, with surgeon preference driving what type of education is provided. Depending on the surgeon, this likely results in significant variance in what patients are taught prior to discharge, although no studies exist in the literature evaluating this.

During our root-cause analysis, patient anxiety surrounding discharge instructions was noted as a predicted barrier to discharge. However, patient-reported anxiety was low both pre- and post-intervention. This perceived anxiety may have, in fact, represented patient's confusion at discharge instructions stemming from a lack of a standardized education regime. Creating video-led education regimes may allow for some standardization of patient education, although the quality of the education must be maintained.

Much of the existing literature surrounding the use of video educational aids in postoperative education focuses on postoperative symptoms, such as pain, where they have been shown to improve patients' pain experience and ability to manage their pain. ${ }^{24-26}$ To our knowledge, there have been no studies assessing the use of video education on streamlining the discharge process and improving discharge times. Thus, our study continues to build on these results, showing that satisfaction can be maintained at a very high level - with most patients rating overall satisfaction 8-10 post-intervention - while simultaneously improving the discharge process.

The use of virtual medical care has been best studied in the outpatient clinical environment, where it has largely been shown to be a safe and effective means of interacting with patients. ${ }^{27}$ A Cochrane review on this topic did not find that there were any increases in morbidity, although the breadth of studies included in this review were limited. ${ }^{27}$ Our patient satisfaction results mirror the results identified in these studies, with high patient satisfaction maintained following implementation of a video education tool. ${ }^{27}$

Late afternoon discharges from a medical or surgical ward can have significant effects in other hospital units, with late afternoon discharges from hospital units being associated with emergency room admission bottlenecks. ${ }^{28}$ In one study assessing patients admitted before or after 1:00 pm, those admitted after 1:00 pm had a risk adjusted increase in their length of stay of 0.6 days. ${ }^{29}$ The approximately $40-\mathrm{min}$ ute mean improvement in discharge time observed in our study may ultimately not be the sole intervention leading to improved discharge times, but perhaps can be one of several interventions that a hospital can use while trying to reach this goal. Although this study focused on one aspect of why late discharges may occur (patient education), there are likely several factors at play. From our own institution's experience, these can occur for simple reasons, such as the lack of a drive home, to administrative issues, such as required paperwork that must be completed prior to discharge. Further study into the specific causes of late discharges will allow targeted strategies to arise.

\section{Strengths and weaknesses}

This is the first reported study to focus on the discharge process and the role that video education can play in structuring this process to allow timely discharge, while still maintaining patient satisfaction. This study was done in a prospective nature, in a practical, real-world fashion, making it applicable to most surgeon's practices. This is the first study to use an outcome measure (discharge time) to track the effect of video education on our patient's clinical experience.

Since patients were not randomized, it is possible that factors other than the intervention contributed to earlier discharge times, such as a change in physician/nursing practice, that would bias our results. Unfortunately, there is no currently available, validated, postoperative satisfaction questionnaire for this patient population, and so our survey may have implicit bias that we are not aware of. Bias may also have been introduced in the voluntary nature of the survey, given the vast majority reporting high satisfaction both preand post-intervention. This could represent a reporting bias, 
with only those satisfied with their care willing to fill out a survey, given the relatively low response rate. We have no reason to believe there is a difference in patients who volunteered to complete the survey before and after the intervention, therefore any bias should affect each group similarly and not impact our conclusions. We did not track physician and nursing time usage pre- and post-intervention, preventing us from assessing if workload was truly reduced. Furthermore, this was not a blinded study, with physicians and nursing teams being aware of the intervention. Although this study tracked discharge time from hospital, no after-hospital assessments were done, preventing us from determining how patients viewed the educational videos once at home.

Several months following the initiation of the intervention, a new electronic medical record (EMR) for the hospital system was instituted. This could have contributed to some of the change in discharge time noted in this study. However, a trend toward improvement was noted prior to the new EMR being used.

\section{Conclusions}

Video postoperative teaching can be a useful adjunct in our armamentarium of tools to improve the discharge process for our patients. This study has shown that video education tools can help with the consistency of patient education, while maintaining high levels of patient satisfaction. Further study is needed to assess the safety of these tools, and whether a shift from in-person education to video education is associated with increased cost (e.g., re-admission rates, increased clinic or emergency room visits post-discharge) or patient morbidity.

Competing interests: The authors do not report any competing personal or financial interests related to this work.

This paper has been peer-reviewed.

\section{References}

1. Stitzenberg KB, Wong YN, Nielsen ME, et al. Trends in radical prostatectomy: Centralization, robotics, and access to urologic cancer care. Cancer 2012;118:54-62. https://doi.org/10.1002/cncr.26274

2. Witherspoon L, Lau JL, Breau RH, et al. Reducing overtreatment of prostate cancer by radical prostatectomy in Eastern Ontario: A population-based cohort study. CMAJ Open 2018;6:E197-201. https://doi.org/10.9778/cmaj0.20170149

3. Bolenz C, Gupta A, Hotze T, et al. Cost comparison of robotic, laparoscopic, and open radical prostatectomy for prostate cancer. Eur Urol 2010;57:453-8. https://doi.org/10.1016/i.eururo.2009.11.008

4. Barbash GI and Glied SA. New technology and healthcare costs: The case of robot-assisted surgery. N Engl $J$ Med 2010;363:701-4. https://doi.org/10.1056/NEJMp1006602

5. Basto M, Sathianathen N, Te Marvelde L, et al. Patterns-of-care and health economic analysis of robot-assisted radical prostatectomy in the Australian public health system. BJU Int 2016;1 17:930-9. https://doi.org/10.1111/bju.13317

6. Hu JC, Gu X, Lipsitz SR, et al. Comparative effectiveness of minimally invasive vs. open radical prostatectomy. J Am Med Assoc 2009;302:1557-64. https://doi.org/10.1001/jama.2009.1451
7. Novara G, Ficarra V, Rosen RC, et al. Systematic review and meta-analysis of perioperative outcomes and complications after robot-assisted radical prostatectomy. Eur. Urol 2012;62:431-52. hitps://doi.org/10.1016/i.eururo.2012.05.044

8. Gandaglia G, Karakiewicz PI, Sun M, et al. Comparative effectiveness of robot-assisted and open radical prostatectomy in the post-dissemination era. J Clin Oncol 2014;32:1419-26. https://doi.org/10.1200/ JC0.2013.53.5096

9. Trinh QD, Sammon J, Sun M, et al. Perioperative outcomes of robot-assisted radical prostatectomy compared with open radical prostatectomy: Results from the nationwide inpatient sample. Eur Urol 2012;61:679-85. https://doi.org/10.1016/i.eururo.2011.12.027

10. Health Quality Ontario: 2011 Quality Improvement Plans: An Analysis for Learning. Published 2011. Available at: httrp://www.hqontario.ca/portals/0/Modals/qi/en/processmap_pdfs/resources_links/ qips-an-analysis-for-learning-2011-en.pdf. Accessed June 22, 2021.

11. Arafeh M, Barghash MA, Haddad N, et al. Using six sigma DMAIC methodology and discrete event simulation to reduce patient discharge time in king hussein cancer center. J Healthc Eng 2018;2018:3832151. https://doi.org/10.1155/2018/3832151

12. Pieper $B$, Sieggreen $M$, Freeland $B$, et al. Discharge information needs of patients after surgery. J Wound Ostomy Continence Nurs 2006;33:281-90. https://doi.org/10.1097/00152192-200605000-00009

13. Love EM, Manalo IF, Chen SC, et al. A video-based educational pilot for basal cell carcinoma (BCC) treatment: A randomized controlled trial. J Am Acad Dermatol 2016;74:477-483.e7. https://doi.org/10.1016/i.jaad.2015.10.014

14. Arpey CJ and Whitaker DC. Postsurgical wound management. Dermatol Clin 2001;19:787-97. https://doi.org/10.1016/S0733-8635(05)70316-8

15. Raine C, Griffiths JM, Potter MA, et al. Written discharge advice sheets reduce visits to the general practitioner. Ann R Coll Surg Engl 1996;78:19-20.

16. Leow JJ, Chang SL, Meyer CP, et al. Robot-assisted versus open radical prostatectomy: A contemporary analysis of an all-payer discharge database. Eur Urol 2016;70:837-45. https://doi.org/10.1016/i. eururo.2016.01.044

17. Toronto: Registered Nurses' Association of Ontario. Nursing Best Practice Guidelines. Available at: http://www.rnao.ca/bpg. Accessed July 10, 2020.

18. Perla RJ, Provost LP and Murray SK. The run chart: A simple analytical tool for learning from variation in healthcare processes. BMJ Qual Saf 2011;20:46-51. https://doi.org/10.1136/bmiqs.2009.037895

19. Moore SM. The effects of a discharge information intervention on recovery outcomes following coronary artery bypass surgery. Int I Nurs Stud 1996;33:181-9. https://doi.org/10.1016/00207489(95)00054-2

20. Pieper $B$, Sieggreen $M$, Nordstrom $C K$, et al. Discharge knowledge and concerns of patients going home with a wound. J Wound Ostomy Cont Nurs 2007;34:245-53;quiz 254-5. https://doi.org/10.1097/01. WON.0000270817.06942.00

21. Albert NM, Buchsbaum R and Li J. Randomized study of the effect of video education on heart failure healthcare utilization, symptoms, and self-care behaviors. Patient Educ Couns 2007;69:129-39. https://doi.org/10.1016/i.pec.2007.08.007

22. Heikkinen K, Leino-Kilpi H, Vahlberg T, et al. Ambulatory orthopaedic surgery patients' symptoms with two different patient education methods. Int I Orthop Trauma Nurs 2012;16:13-20. https://doi.org/10.1016/i.ijotn.2011.04.004

23. Lovell MR, Luckett T, Boyle FM, et al. Patient education, coaching, and self-management for cancer pain. J Clin Oncol 2014;16:1712-20. https://doi.org/10.1200/JC0.2013.52.4850

24. Mosso-Vázquez JL, Gao K, Wiederhold BK, et al. Virtual reality for pain management in cardiac surgery. Cyberpsychology Behav Soc Netw 2014;17:371-8. https://doi.org/10.1089/cyber.2014.0198

25. Martorella G, Côté J and Choinière M. SOULAGE-TAVIE: Development and validation of a virtual nursing intervention to promote self-management of postoperative pain after cardiac surgery. CIN Comput Inform Nurs 2013;31:189-97. hittps://doi.org/10.1097/NXN.0b013e3182812d69

26. Kipping B, Rodger $S$, Miller $K$, et al. Virtual reality for acute pain reduction in adolescents undergoing burn wound care: A prospective, randomized controlled trial. Burns 2012;38:650-7. https://doi.org/10.1016/i.burns.2011.11.010

27. Flodgren $G$, Rachas $A$, Farmer AJ, et al. Interactive telemedicine: effects on professional practice and health care outcomes. Cochrane Database Syst Rev 2015;2015CD002098. https://doi.org/10.1002/14651858.CD002098.pub2

28. Khanna S, Boyle J, Good N, et al. Impact of admission and discharge peak times on hospital overcrowding. Stud Health Technol Inform 2011;168:82-8.

29. Wertheimer B, Jacobs REA, Bailey M, et al. Discharge before noon: An achievable hospital goal. J Hosp Med 2014;9:210-4. https://doi.org/10.1002/ihm.2154

Correspondence: Dr. Luke D.E. Witherspoon, Division of Urology, The University of Ottawa, Ottawa, ON, Canada; Iwitherspoon@toh.ca 